\title{
Putative Malignant Pleural Mesothelioma in situ (MPMIS) with Sequential Acquisition of Genomic Alterations on Fluorescence in situ Hybridization (FISH) Examination
}

\author{
Simon Haefliger ${ }^{\mathrm{a}}$ Spasenija Savice Prince ${ }^{\mathrm{a}} \quad$ Julien Rebetez ${ }^{\mathrm{a}} \quad$ Heinz Borer $^{\mathrm{b}}$ \\ Lukas Bubendorfa
}

aDepartment of Pathology, Institute of Medical Genetics and Pathology, University Hospital of Basel, University of Basel, Basel, Switzerland; 'bepartment of Pulmonology, Bürgerspital Solothurn, Solothurn, Switzerland

\section{Established Facts}

- Criteria for the diagnosis of malignant pleural mesothelioma in situ have been proposed to be based on clinical, radiological, and morphological features.

- Fluorescence in situ hybridization analysis is a sensitive and highly specific ancillary tool for the diagnosis of malignant mesothelial cells.

- Satellitosis is a morphological phenomenon described among others in the context of acute alcoholic hepatitis.

\section{Novel Insights}

- Fluorescence in situ hybridization analysis and BAP1 immunochemistry are a potential screening tools to detect malignant pleural mesothelioma in situ in patients presenting with recurrent effusions of unknown origin and no clinical evidence of mesothelioma.

- Genomic transition from a diploid to an aneuploid state might play a role in progression from mesothelioma in situ to invasive mesothelioma.

- Satellitosis of mesothelial cells by lymphocytes in pleural effusion cytology is a rare and not tumorspecific phenomenon with unclear relevance.

\section{Keywords}

Mesothelioma in situ · BAP1 · CDKN2A - 9p21 deletion ·

Satellitosis

\section{Abstract}

Malignant pleural mesothelioma (MPM) is a rare and deadly disease. A precursor in situ lesion, malignant pleural meso- thelioma in situ (MPMIS), has recently been proposed. On cytological examination, the distinction between reactive and malignant mesothelial cells is often challenging, and sometimes even impossible without ancillary methods. Fluorescence in situ hybridization (FISH) for detection of 9p21 deletion is a powerful diagnostic tool in this context, both in histological and in cytological specimens. Here, we present a case of MPM with initial presentation as a putative MPMIS
(C) 2020 The Author(s)

Published by S. Karger AG, Basel

This is an Open Access article licensed under the Creative Commons Attribution-NonCommercial-4.0 International License (CC BY-NC) (http://www.karger.com/Services/OpenAccessLicense), applicable to the online version of the article only. Usage and distribution for commercial purposes requires written permission.
Lukas Bubendorf

Institute of Pathology and Medical Genetics, University Hospital Basel Schönbeinstrasse 40

$\mathrm{CH}-4031$ Basel (Switzerland)

Lukas.Bubendorf@usb.ch 
with disomic chromosomal pattern and homozygous 9p21 deletion with subsequent development of an aneuploid pattern after whole genome duplication during tumor progression.

(C) 2020 The Author(s)

Published by S. Karger AG, Basel of an established malignant mesothelioma. Here, we present a case of MPM with initial presentation as a putative in situ lesion showing sequential acquisition of genomic alterations on fluorescence in situ hybridization (FISH) examination during tumor progression.

\section{Case Presentation}

A 57-year-old man, non-smoker, and electro-mechanic by profession, presented with cough and B-symptoms for more than 3 months. Clinically and radiologically, a MPM was suspected. A thoracentesis was performed, and pleural fluid was obtained. On cytological examination, there were numerous malignant mesothelial-like cells with frank atypia (shown in Fig. 1a). The mesothelial phenotype was confirmed by immunochemistry (IC) and showed calretinin expression and absence of BerEP4 expression. There was a complete loss of desmin expression. The diagnosis of malignant mesothelial cells was ascertained by FISH using a
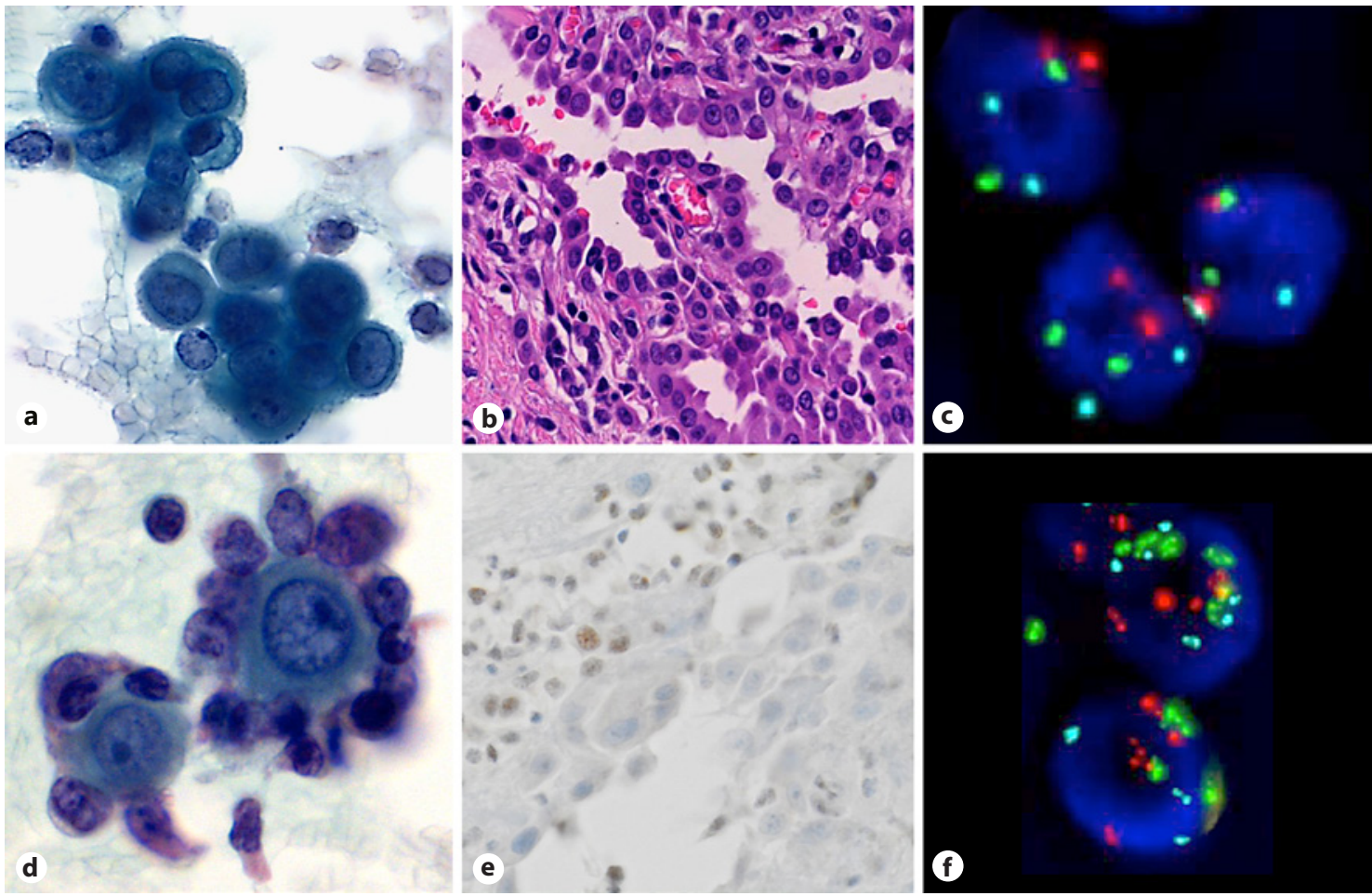

Fig. 1. a Pleural effusion at the time of diagnosis showing large clusters of unequivocal malignant mesothelial cells with prominent nucleoli (Papanicolaou [Pap] stain, ×630). d Initial pleural effusion highlighting satellitosis of mesothelial cells by lymphocytes; the mesothelial cells only show mild atypia (Pap stain, $\times 630$ ). b, e Initial pleural biopsy: atypical mesothelial cells on histology (b, hematoxylin and eosin stain, $\times 400$ ) with loss of BAP1 expression. Internal positive control with nuclear BAP1 expression is observed in the adjacent leucocytes (e, immunoperoxidase, $\times 400$ ). c Multiprobe FISH result of the initial effusion: regular disomic pattern for chromosomes 3 (red), 7 (green), and 17 (blue) and homozygous deletion of the chromosomal region 9p21 (lack of gold signal) $(\times 630)$. $f$ Multiprobe FISH result of the pleural biopsy of malignant mesothelioma 8 months later: homozygous deletion of the chromosomal region 9p21 (lack of gold signal) and polysomy of the chromosome 3 (red), 7 (green), and 17 (blue) in the cytological specimen (f, DAPI). FISH, fluorescence in situ hybridization. 
multiprobe FISH assay (UroVysion ${ }^{\mathrm{TM}}$, Abbott, Chicago, IL, USA) revealing unbalanced polysomy of the chromosomes 3,7 , and 17 and homozygous loss of 9p21 (shown in Fig. 1f). The MPM diagnosis was confirmed by a subsequent pleural biopsy. Interestingly, 8 months before, we had received pleural effusion fluid and a pleural biopsy from the patient, which had been diagnosed as unspecific organizing pleuritis without signs of malignancy (shown in Fig. 1b, 2a-b). At this time, no mesothelioma was suspected, radiologically and clinically. Review of the biopsy samples showed single layered and mildly atypical mesothelial cells without infiltrative growth pattern. Also, the effusion cytology has been rendered as unsuspicious. On review, it contained lymphocytes and mesothelial cells without frank atypia. Interestingly, a substantial number of the mesothelial cells were surrounded and encircled by lymphocytes, which we refer to as satellitosis of mesothelial cells by lymphocytes (shown in Fig. 1d). The encircled mesothelial cells were positive for calretinin. Satellitosis of mesothelial cells by lymphocytes was not present in the cytological specimen with the full-blown mesothelioma. Retrospective IC and FISH performed at the initial presentation revealed a loss of desmin and BAP1 expression (shown in Fig. 1e) as well as a homozygous deletion of 9p21 (shown in Fig. 1c) but without polysomy of the chromosome 3,7 , and 17 as seen in the subsequent effusion specimen with MPM diagnosis (shown in Fig. 1f). Considering these results, we hypothesize that during the time interval between the 2 samples ( 8 months), the tumor cells had undergone whole genome duplication and that the lesion initially biopsied could represent an in situ form of MPM as, at this time, there was no radiological and clinical evidence of MPM. A pleural biopsy confirmed the diagnosis of mesothelioma (shown in Fig. 2c), and a loss of MTAP could be observed on retrospective IHC (shown in Fig. $2 \mathrm{~d}-\mathrm{e}$ ). Unfortunately, there was no more tissue left for MTAP IHC of the early manifestation after various IHC and FISH analyses.

Being intrigued by the satellitosis of mesothelial cells by lymphocytes in the initial specimen, we performed a data base search for Papanicolaou-stained cytological samples containing "malignant pleural mesothelioma" and "lymphocyte-rich effusion." These specimens were reviewed in order to determine the incidence of this phenomenon in pleural effusions. We identified 60 cases with either histological or radiological proven MPM and reviewed 60 cases with the diagnosis of "reactive mesothelial cells" and "lymphocyte-rich effusion." We observed "satellitosis" in a total of 5 cases, 2 cases with malignant mesothelial cells including the case presented here, and 3 cases with reactive mesothelial cells (shown in Fig. 3a-d).

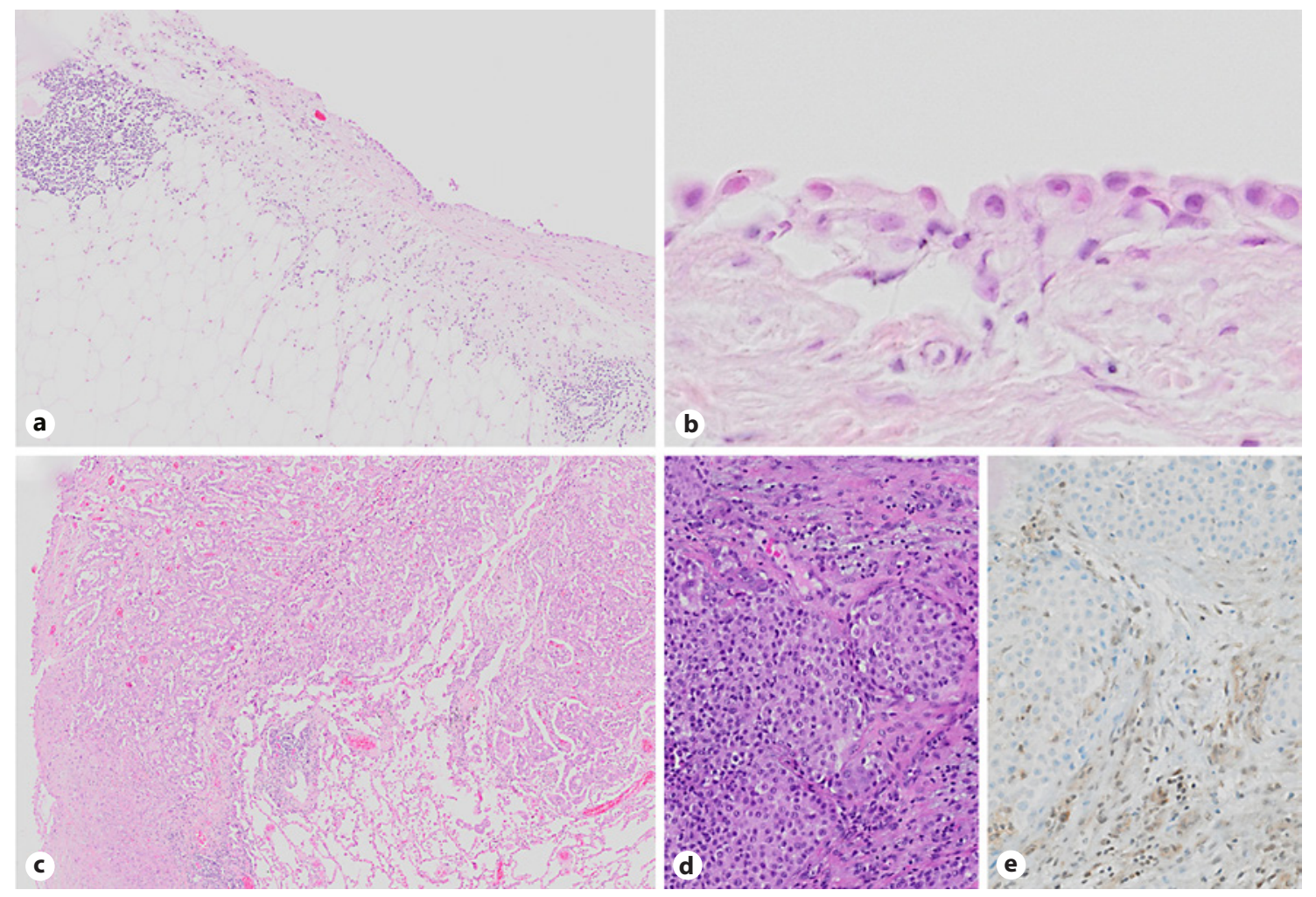

Fig. 2. a Low-power view of the initial pleural biopsy being consistent with chronic pleuritis with lymphocytic inflammation (a, HE, $\times 5$ ). b High-power view of the initial pleural biopsy, highlighting a single layer of cuboidal mesothelial cells, without frank atypia at the surface of the pleura $(\mathbf{b}, \mathrm{HE}, \times 20)$. c Low-power view of the invasive pleural mesothelioma with infiltration of the lung parenchyma $(\mathbf{c}, \mathrm{HE}, \times 5)$. d, e Complete loss of MTAP in the neoplastic mesothelial cells by IHC. Retained MTAP expression in adjacent lymphocytes and stromal cells $(\mathbf{d}, \mathbf{e}, \mathrm{HE}, \times 20)$. 
Fig. 3. a, b Conventional cytology with benign mesothelial cells surrounded by lymphocytes satellitosis of mesothelial cells by lymphocytes (a, b, ×630, Papanicolaou stain). c, d The mesothelial nature of the encircled cells from our patient at the time of the putative MPMIS was confirmed by immunocytochemistry for calretinin $(\mathbf{c}, \mathbf{d}$, $\times 630$, immunoperoxidase).

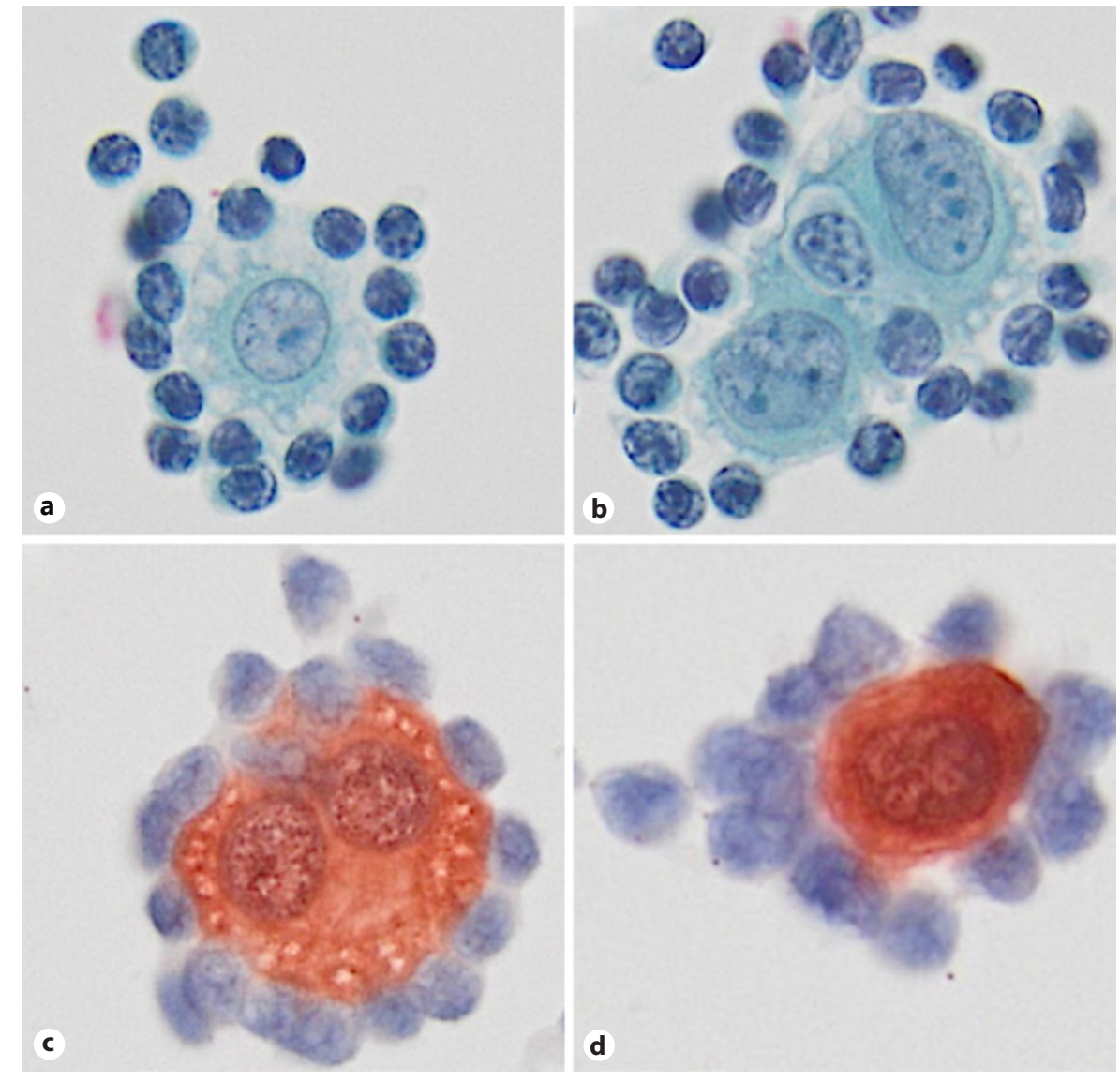

\section{Discussion and Conclusion}

The existence of an in situ precursor of invasive malignant mesothelioma has long been disputed but is now an accepted diagnostic concept [2-4]. The main challenge in clinical practice lies in the large surface of the pleura, which makes it impossible to exclude coexistent focally invasive MPM due to limited biopsy sampling. The International Association for the Study of Lung Cancer recently proposed that MPMIS lesions should be diagnosed based on clinical, radiological, and morphological features, the prerequisite being the absence of clinical and radiological evidence of mesothelioma [3]. In this setting, an atypical mesothelial proliferation limited to the pleural surface only qualifies as MPMIS if a $B R C A 1$ associated protein-1 (BAP1) loss and/or cyclin-dependent kinase inhibitor $2 \mathrm{~A}(C D K N 2 A)$ homozygous deletion is present [3, 4]. In our case, the neoplastic mesothelial cells present in the pleura sample at the time of the initial effusion meet the International Association for the Study of Lung Cancer criteria proposed for MPMIS, as the patient did not show any radiologic evidence of mesothelioma and as the mesothelial proliferation was restricted to the pleural surface with homozygous deletion of $9 \mathrm{p} 21$ containing the $C D K N 2 A$ gene and loss of BAP1 expression.

FISH analysis has been shown to be a sensitive and highly specific ancillary tool for the diagnosis of malignant mesothelial cells and for its distinction from reactive mesothelial cells in histological biopsies and effusion cytology, respectively [5-7]. In the present case, FISH played a key role in identifying the mesothelial cells as malignant both at the time of the first effusion and at the time of clinically manifest disease 8 months later. Using a multiprobe FISH assay also allowed us to observe genomic progression from a diploid to an aneuploid state, which was most likely driven by whole genome doubling. Whole genome doubling has recently been recognized as a macro-evolutionary event in cancer arising early in carcinogenesis after an antecedent transforming driver mutation [8]. Homozygous 9p21 deletion and/or inactivating BAP1 mutation could qualify as such early transforming alterations in case of malignant mesothelioma. The combina- 
tion of BAP1 IC and FISH for detection of 9p21 deletion has been shown to be complementary to increase the sensitivity for diagnosing MPM [9]. In addition, loss of MTAP expression by IC has emerged as a promising surrogate marker for homozygous 9 p21 deletion, which is particularly interesting for laboratories without access to FISH analysis [7, 10]. The MTAP gene is located near to $C D K N 2 A / p 16$ and co-deleted in the vast majority of MPM with 9p21 deletion. However, until there are more data on the robustness of MTAP IC, FISH remains the gold standard to detect homozygous 9p21 deletions. The rapid progression from invisible early diploid mesothelial neoplasia to an advanced aneuploid manifestation of diffuse mesothelioma within a short time interval of 8 months would be remarkable, but possible considering the variable progression dynamics of pleural mesothelio$\mathrm{ma}$ [8]. The interval between in situ and invasive mesothelioma have been as short as 12 months [8]. Nevertheless, we cannot exclude an undetected focus of established aneuploid mesothelioma at the time of the first effusion due to limited sampling.

Even if the main cytological features that indicate a mesothelial origin for neoplastic cells are well described, in practice, the diagnosis of malignant mesothelioma can be difficult [11]. Sometimes, the malignant cells are indistinguishable from benign, reactive mesothelial cells, as evidenced by the first effusion of our patient [12]. The intriguing satellitosis of the rare neoplastic mesothelial cells by lymphocytes could be suspected to reflect early interaction of T-cells due to neoantigen recognition. Interestingly, it has previously been proposed that human peritoneal mesothelial cells are equipped with an antigen-presenting machinery to recall antigens to T-cells [13-15]. The observed satellitosis might be a morphological correlate of such interaction between T-cells and mesothelial cells. Satellitosis - defined as abnormal clustering of cells encircling others cells - has been observed in the context of acute alcoholic hepatitis, where ballooned, damaged hepatocytes are surrounded by neutrophils or in certain brain tumors, for example, in oligodendroglioma, where the neoplastic oligodendrocytes tend to cluster around neurons exhibiting a so called "perineuronal satellitosis" $[16,17]$. Our review of a consecutive series of benign and malignant mesothelioma effusions showed that satellitosis of mesothelial cells by lymphocytes is a rare phenomenon that is not specific for malignant mesothelial as we observed it in both reactive and malignant samples. We cannot exclude that this phenomenon only represents a physical artifact caused by unknown variables during processing of the samples. Nevertheless, further investiga-

A Case of a Putative Malignant Pleural

Mesothelioma in situ tions are needed to study if there are specific interactions between lymphocytes and mesothelial cells in reactive conditions or during early mesothelial oncogenesis that could explain this morphological finding.

In conclusion, we present a case of putative MPMIS with homozygous 9p21 deletion and diploid chromosomal pattern that progressed to a clinically manifest aneuploid MPM as shown by FISH analysis. This report emphasizes the utility of ancillary testing to detect MPMIS or early invasive MPM even in cytology or biopsy specimens that would otherwise be diagnosed as benign or reactive in patients presenting with recurrent effusions of unknown origin. It also illustrates the potential usefulness of ancillary testing by FISH analysis and/or BAP1 IC as screening tools for MPMIS in patients at risk due to a history of asbestos exposure who present with otherwise unexplained pleural effusion. Satellitosis of mesothelial cells by lymphocytes is a rare and not tumor-specific phenomenon with unclear relevance.

\section{Acknowledgement}

We would like to thank Dr. med. Christine Egger for providing us with material for immunohistochemical analysis.

\section{Statement of Ethics}

This study was covered by the local institutional approval EK 253/08 on human research (EKBB/EKNZ). A written informed consent could not be obtained from the patient as he passed away 10 years ago. As the report does not contain any critical data (picture or radiography of the patient) and consequently anonymity is guaranteed, a consent from the patient is, in this particular case, not strictly required according to the ethics approval.

\section{Conflict of Interest Statement}

The authors have no conflict of interest to declare.

\section{Funding Sources}

The authors have no funding to declare.

\section{Author Contributions}

L.B. and S.H. conceived and designed the project. L.B., S.S., J.R., and S.H. analyzed the data. H.B. obtained cytological and histological specimens. S.H., L.B., H.B., and S.S. wrote the manuscript. All authors agreed to the content of the manuscript. 


\section{References}

1 Yap TA, Aerts JG, Popat S, Fennell DA. Novel insights into mesothelioma biology and implications for therapy. Nat Rev Cancer. 2017 Jul;17(8):475-88.

2 Churg A, Hwang H, Tan L, Qing G, Taher A, Tong A, et al. Malignant mesothelioma in situ. Histopathology. 2018 May;72(6):10338.

3 Nicholson AG, Sauter JL, Nowak AK, Kindler HL, Gill RR, Remy-Jardin M, et al. EURACAN/IASLC proposals for updating the histologic classification of pleural mesothelioma: towards a more multidisciplinary approach. J Thorac Oncol. 2020 Jan;15(1):29-49.

4 Churg A, Galateau-Salle F, Roden AC, Attanoos R, von der Thusen JH, Tsao MS, et al. Malignant mesothelioma in situ: morphologic features and clinical outcome. Mod Pathol. 2020;33(2):297-302.

5 Savic S, Franco N, Grilli B, Barascud AV, Her$\operatorname{zog} \mathrm{M}$, Bode B, et al. Fluorescence in situ hybridization in the definitive diagnosis of malignant mesothelioma in effusion cytology. Chest. 2010 Jul;138(1):137-44.

6 Chiosea S, Krasinskas A, Cagle PT, Mitchell KA, Zander DS, Dacic S. Diagnostic importance of 9p21 homozygous deletion in malignant mesotheliomas. Mod Pathol. 2008 Jun; 21(6):742-7.
7 Hamasaki M, Kinoshita Y, Yoshimura M, Matsumoto S, Kamei T, Hiroshima K, et al. Cytoplasmic MTAP expression loss detected by immunohistochemistry correlates with 9p21 homozygous deletion detected by FISH in pleural effusion cytology of mesothelioma. Histopathology. 2019 Jul;75(1):153-5.

8 Bielski CM, Zehir A, Penson AV, Donoghue MTA, Chatila W, Armenia J, et al. Genome doubling shapes the evolution and prognosis of advanced cancers. Nat Genet. 2018 Aug. 50(8):1189-95.

9 Sheffield BS, Hwang HC, Lee AF, Thompson $\mathrm{K}$, Rodriguez S, Tse $\mathrm{CH}$, et al. BAP1 immunohistochemistry and p16 FISH to separate benign from malignant mesothelial proliferations. Am J Surg Pathol. 2015 Jul;39(7):97782.

10 Chapel DB, Schulte JJ, Berg K, Churg A, Dacic S, Fitzpatrick C, et al. MTAP immunohistochemistry is an accurate and reproducible surrogate for CDKN2A fluorescence in situ hybridization in diagnosis of malignant pleural mesothelioma. Mod Pathol. 2020 Feb; 33(2):245-54.

11 Hjerpe A, Abd-Own S, Dobra K. Cytopathologic diagnosis of epithelioid and mixed-type malignant mesothelioma: ten years of clinical experience in relation to international guidelines. Arch Pathol Lab Med. 2018 Aug;142(8): 893-901.
12 Sherman ME, Mark EJ. Effusion cytology in the diagnosis of malignant epithelioid and biphasic pleural mesothelioma. Arch Pathol Lab Med. 1990 Aug;114(8):845-51.

13 Shaw TJ, Zhang XY, Huo Z, Robertson D, Lovell PA, Dalgleish AG, et al. Human peritoneal mesothelial cells display phagocytic and antigen-presenting functions to contribute to intraperitoneal immunity. Int J Gynecol Cancer. 2016 Jun;26(5):833-8.

14 Gjersten MK, Saeterdal I, Beiske K, Gaudernack $\mathrm{G}$. Antigen-presenting function of human peritoneum mesothelial cells isolated from a pancreatic carcinoma patient after mutant Ras peptide vaccination. Cancer Immunol Immunother. 1996 Dec;43(5):262-8.

15 Hausmann MJ, Rogachev B, Weiler M, Chaimovitz C, Douvdevani A. Accessory role of human peritoneal mesothelial cells in antigen presentation and T-cell growth. Kidney Int. $2000 \mathrm{Feb} ; 57(2): 476-86$.

16 Lefkowitch JH. Morphology of alcoholic liver disease. Clin Liver Dis. 2005 Feb;9(1):37-53.

17 Wesseling P, van den Bent M, Perry A. Oligodendroglioma: pathology, molecular mechanisms and markers. Acta Neuropathol. 2015 Jun;129(6):809-27. 